\title{
Pretrained Transformers Improve Out-of-Distribution Robustness
}

\author{
Dan Hendrycks ${ }^{1 *}$ \\ Xiaoyuan Liu $^{1,2 *}$ \\ Eric Wallace ${ }^{1}$ \\ Adam Dziedzic $^{3}$ \\ Rishabh Krishnan ${ }^{1}$ \\ Dawn Song ${ }^{1}$ \\ ${ }^{1}$ UC Berkeley ${ }^{2}$ Shanghai Jiao Tong University ${ }^{3}$ University of Chicago \\ \{hendrycks,ericwallace, dawnsong\} @berkeley.edu
}

\begin{abstract}
Although pretrained Transformers such as BERT achieve high accuracy on indistribution examples, do they generalize to new distributions? We systematically measure out-of-distribution (OOD) generalization for seven NLP datasets by constructing a new robustness benchmark with realistic distribution shifts. We measure the generalization of previous models including bag-of-words models, ConvNets, and LSTMs, and we show that pretrained Transformers' performance declines are substantially smaller. Pretrained transformers are also more effective at detecting anomalous or OOD examples, while many previous models are frequently worse than chance. We examine which factors affect robustness, finding that larger models are not necessarily more robust, distillation can be harmful, and more diverse pretraining data can enhance robustness. Finally, we show where future work can improve OOD robustness.
\end{abstract}

\section{Introduction}

The train and test distributions are often not identically distributed. Such train-test mismatches occur because evaluation datasets rarely characterize the entire distribution (Torralba and Efros, 2011), and the test distribution typically drifts over time (Quionero-Candela et al., 2009). Chasing an evolving data distribution is costly, and even if the training data does not become stale, models will still encounter unexpected situations at test time. Accordingly, models must generalize to OOD examples whenever possible, and when OOD examples do not belong to any known class, models must detect them in order to abstain or trigger a conservative fallback policy (Emmott et al., 2015).

Most evaluation in natural language processing (NLP) assumes the train and test examples are in-

\footnotetext{
${ }^{*}$ Equal contribution.

https://github.com/camelop/NLP-Robustness
}

dependent and identically distributed (IID). In the IID setting, large pretrained Transformer models can attain near human-level performance on numerous tasks (Wang et al., 2019). However, high IID accuracy does not necessarily translate to OOD robustness for image classifiers (Hendrycks and Dietterich, 2019), and pretrained Transformers may embody this same fragility. Moreover, pretrained Transformers can rely heavily on spurious cues and annotation artifacts (Cai et al., 2017; Gururangan et al., 2018) which out-of-distribution examples are less likely to include, so their OOD robustness remains uncertain.

In this work, we systematically study the OOD robustness of various NLP models, such as word embeddings averages, LSTMs, pretrained Transformers, and more. We decompose OOD robustness into a model's ability to (1) generalize and to (2) detect OOD examples (Card et al., 2018).

To measure OOD generalization, we create a new evaluation benchmark that tests robustness to shifts in writing style, topic, and vocabulary, and spans the tasks of sentiment analysis, textual entailment, question answering, and semantic similarity. We create OOD test sets by splitting datasets with their metadata or by pairing similar datasets together (Section 2). Using our OOD generalization benchmark, we show that pretrained Transformers are considerably more robust to OOD examples than traditional NLP models (Section 3). We show that the performance of an LSTM semantic similarity model declines by over $35 \%$ on OOD examples, while a RoBERTa model's performance slightly increases. Moreover, we demonstrate that while pretraining larger models does not seem to improve OOD generalization, pretraining models on diverse data does improve OOD generalization.

To measure OOD detection performance, we turn classifiers into anomaly detectors by using their prediction confidences as anomaly scores 
(Hendrycks and Gimpel, 2017). We show that many non-pretrained NLP models are often near or worse than random chance at OOD detection. In contrast, pretrained Transformers are far more capable at OOD detection. Overall, our results highlight that while there is room for future robustness improvements, pretrained Transformers are already moderately robust.

\section{How We Test Robustness}

\subsection{Train and Test Datasets}

We evaluate OOD generalization with seven carefully selected datasets. Each dataset either (1) contains metadata which allows us to naturally split the samples or (2) can be paired with a similar dataset from a distinct data generating process. By splitting or grouping our chosen datasets, we can induce a distribution shift and measure OOD generalization. We utilize four sentiment analysis datasets:

- We use SST-2, which contains pithy expert movie reviews (Socher et al., 2013), and IMDb (Maas et al., 2011), which contains fulllength lay movie reviews. We train on one dataset and evaluate on the other dataset, and vice versa. Models predict a movie review's binary sentiment, and we report accuracy.

- The Yelp Review Dataset contains restaurant reviews with detailed metadata (e.g., user ID, restaurant name). We carve out four groups from the dataset based on food type: American, Chinese, Italian, and Japanese. Models predict a restaurant review's binary sentiment, and we report accuracy.

- The Amazon Review Dataset contains product reviews from Amazon (McAuley et al., 2015; He and McAuley, 2016). We split the data into five categories of clothing (Clothes, Women Clothing, Men Clothing, Baby Clothing, Shoes) and two categories of entertainment products (Music, Movies). We sample 50,000 reviews for each category. Models predict a review's 1 to 5 star rating, and we report accuracy.

We also utilize these datasets for semantic similarity, reading comprehension, and textual entailment:

- STS-B requires predicting the semantic similarity between pairs of sentences (Cer et al., 2017). The dataset contains text of different genres and sources; we use four sources from two genres: MSRpar (news), Headlines (news); MSRvid (captions), Images (captions). The evaluation metric is Pearson's correlation coefficient.
- ReCoRD is a reading comprehension dataset using paragraphs from CNN and Daily Mail news articles and automatically generated questions (Zhang et al., 2018). We bifurcate the dataset into CNN and Daily Mail splits and evaluate using exact match.

- MNLI is a textual entailment dataset using sentence pairs drawn from different genres of text (Williams et al., 2018). We select examples from two genres of transcribed text (Telephone and Face-to-Face) and one genre of written text (Letters), and we report classification accuracy.

\subsection{Embedding and Model Types}

We evaluate NLP models with different input representations and encoders. We investigate three model categories with a total of thirteen models.

Bag-of-words (BoW) Model. We use a bag-ofwords model (Harris, 1954), which is high-bias but low-variance, so it may exhibit performance stability. The BoW model is only used for sentiment analysis and STS-B due to its low performance on the other tasks. For STS-B, we use the cosine similarity of the BoW representations from the two input sentences.

Word Embedding Models. We use word2vec (Mikolov et al., 2013) and GloVe (Pennington et al., 2014) word embeddings. These embeddings are encoded with one of three models: word averages (Wieting et al., 2016), LSTMs (Hochreiter and Schmidhuber, 1997), and Convolutional Neural Networks (ConvNets). For classification tasks, the representation from the encoder is fed into an MLP. For STS-B and MNLI, we use the cosine similarity of the encoded representations from the two input sentences. For reading comprehension, we use the DocQA model (Clark and Gardner, 2018) with GloVe embeddings. We implement our models in AllenNLP (Gardner et al., 2018) and tune the hyperparameters to maximize validation performance on the IID task.

Pretrained Transformers. We investigate BERT-based models (Devlin et al., 2019) which are pretrained bidirectional Transformers (Vaswani et al., 2017) with GELU (Hendrycks and Gimpel, 2016) activations. In addition to using BERT Base and BERT Large, we also use the large version of RoBERTa (Liu et al., 2019b), which is pretrained on a larger dataset than BERT. 
Semantic Textual Similarity (STS-B) Generalization

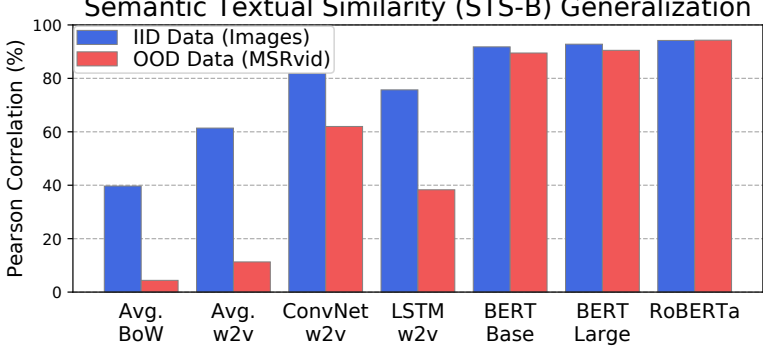

Figure 1: Pretrained Transformers often have smaller IID/OOD generalization gaps than previous models.

We use ALBERT (Lan et al., 2020) and also a distilled version of BERT, DistilBERT (Sanh et al., 2019). We follow the standard BERT fine-tuning procedure (Devlin et al., 2019) and lightly tune the hyperparameters for our tasks. We perform our experiments using the HuggingFace Transformers library (Wolf et al., 2019).

\section{Out-of-Distribution Generalization}

In this section, we evaluate OOD generalization of numerous NLP models on seven datasets and provide some upshots. A subset of results are in Figures 1 and 2. Full results are in the Appendix.

Pretrained Transformers are More Robust. In our experiments, pretrained Transformers often have smaller generalization gaps from IID data to OOD data than traditional NLP models. For instance, Figure 1 shows that the LSTM model declined by over $35 \%$, while RoBERTa's generalization performance in fact increases. For Amazon, MNLI, and Yelp, we find that pretrained Transformers' accuracy only slightly fluctuates on OOD examples. Partial MNLI results are in Table 1. We present the full results for these three tasks in the Appendix. In short, pretrained Transformers can generalize across a variety of distribution shifts.

\begin{tabular}{c|ccc} 
Model & $\begin{array}{c}\text { Telephone } \\
\text { (IID) }\end{array}$ & $\begin{array}{c}\text { Letters } \\
\text { (OOD) }\end{array}$ & $\begin{array}{c}\text { Face-to-Face } \\
\text { (OOD) }\end{array}$ \\
\hline BERT & $81.4 \%$ & $82.3 \%$ & $80.8 \%$ \\
\hline
\end{tabular}

Table 1: Accuracy of a BERT Base MNLI model trained on Telephone data and tested on three different distributions. Accuracy only slightly fluctuates.

Bigger Models Are Not Always Better. While larger models reduce the IID/OOD generalization gap in computer vision (Hendrycks and $\mathrm{Di}$ etterich, 2019; Xie and Yuille, 2020; Hendrycks et al., 2019d), we find the same does not hold in NLP. Figure 3 shows that larger BERT and AL-

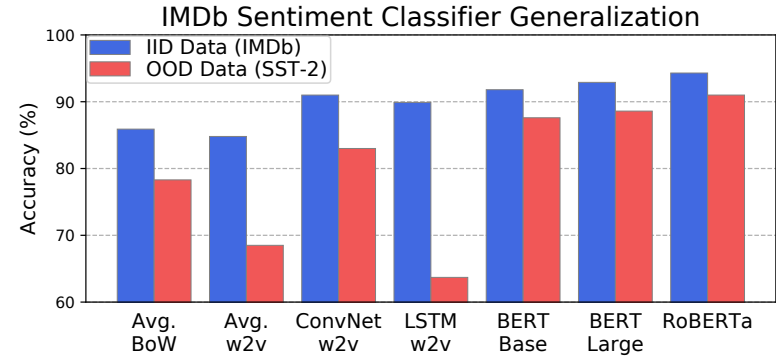

ReCoRD Reading Comprehension Generalization

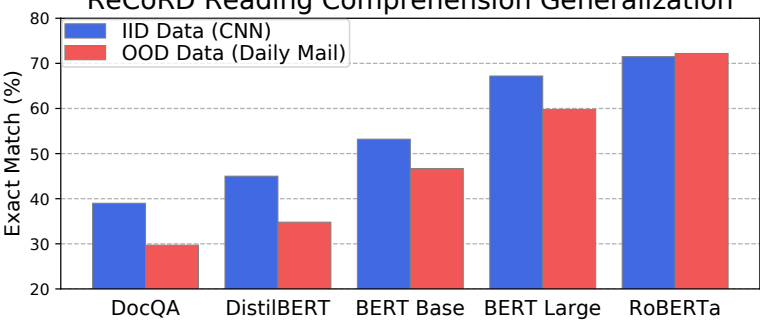

Figure 2: Generalization results for sentiment analysis and reading comprehension. While IID accuracy does not vary much for IMDb sentiment analysis, OOD accuracy does. Here pretrained Transformers do best.

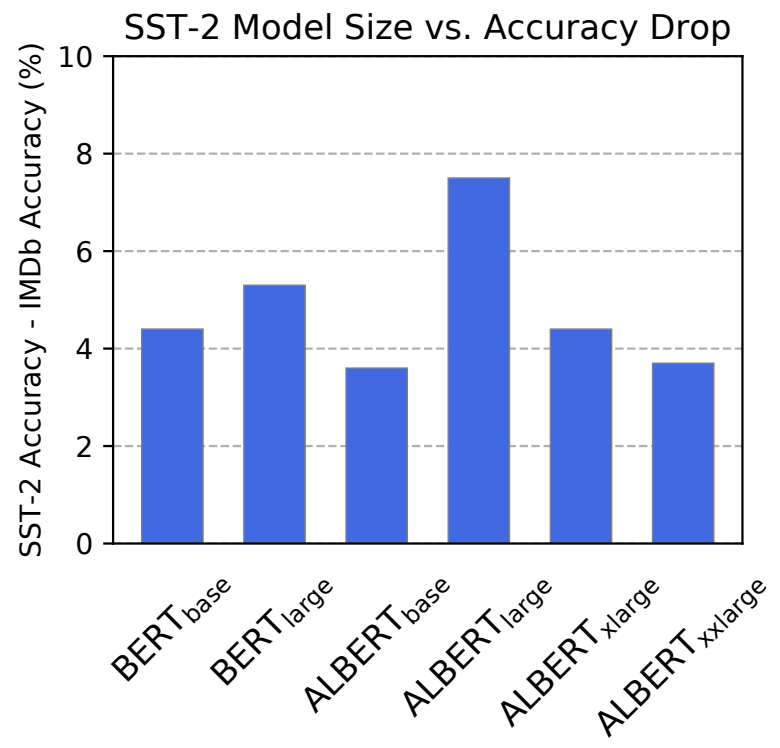

Figure 3: The IID/OOD generalization gap is not improved with larger models, unlike in computer vision.

BERT models do not reduce the generalization gap. However, in keeping with results from vision (Hendrycks and Dietterich, 2019), we find that model distillation can reduce robustness, as evident in our DistilBERT results in Figure 2. This highlights that testing model compression methods for BERT (Shen et al., 2020; Ganesh et al., 2020; Li et al., 2020) on only in-distribution examples gives a limited account of model generalization, and such narrow evaluation may mask downstream costs.

More Diverse Data Improves Generalization. Similar to computer vision (Orhan, 2019; Xie et al., 


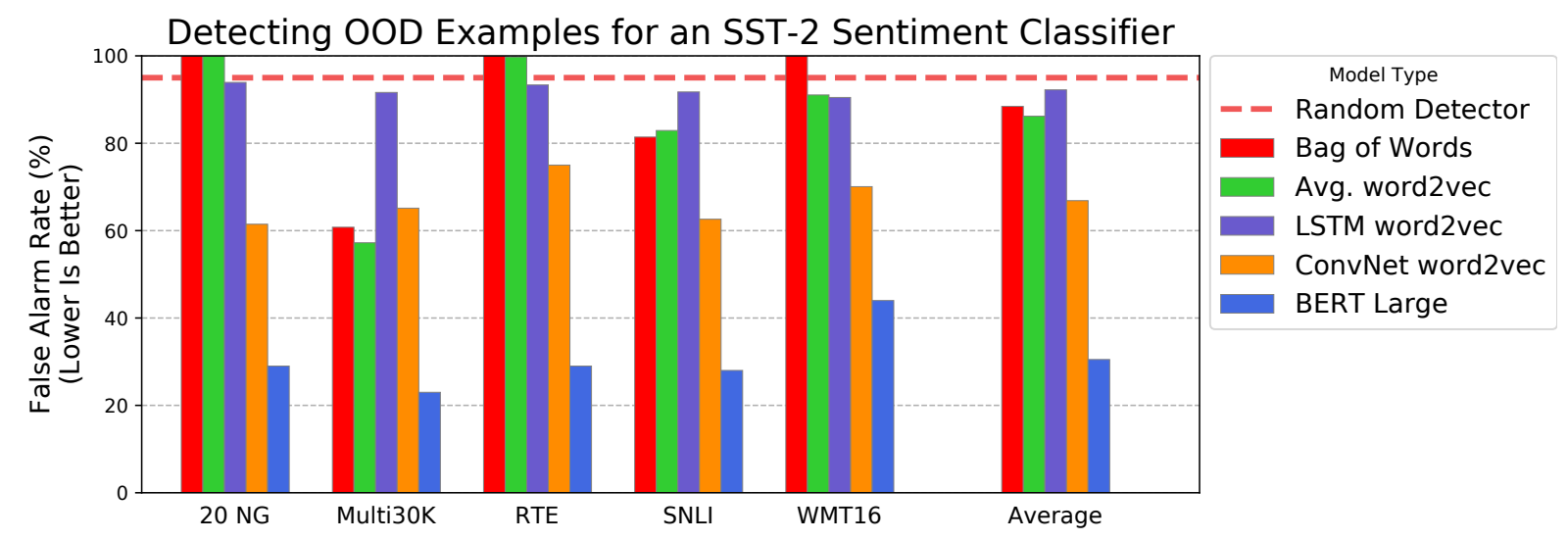

Figure 4: We feed in OOD examples from out-of-distribution datasets (20 Newsgroups, Multi30K, etc.) to SST-2 sentiment classifiers and report the False Alarm Rate at 95\% Recall. A lower False Alarm Rate is better. Classifiers are repurposed as anomaly detectors by using their negative maximum softmax probability as the anomaly scoreOOD examples should be predicted with less confidence than IID examples. Models such as BoW, word2vec averages, and LSTMs are near random chance; that is, previous NLP models are frequently more confident when classifying OOD examples than when classifying IID test examples.

2020; Hendrycks et al., 2019a), pretraining on larger and more diverse datasets can improve robustness. RoBERTa exhibits greater robustness than BERT Large, where one of the largest differences between these two models is that RoBERTa pretrains on more data. See Figure 2's results.

\section{Out-of-Distribution Detection}

Since OOD robustness requires evaluating both OOD generalization and OOD detection, we now turn to the latter. Without access to an outlier dataset (Hendrycks et al., 2019b), the state-ofthe-art OOD detection technique is to use the model's prediction confidence to separate in- and out-of-distribution examples (Hendrycks and Gimpel, 2017). Specifically, we assign an example $x$ the anomaly score $-\max _{y} p(y \mid x)$, the negative prediction confidence, to perform OOD detection.

We train models on SST-2, record the model's confidence values on SST-2 test examples, and then record the model's confidence values on OOD examples from five other datasets. For our OOD examples, we use validation examples from 20 Newsgroups (20 NG) (Lang, 1995), the English source side of English-German WMT16 and English-German Multi30K (Elliott et al., 2016), and concatenations of the premise and hypothesis for RTE (Dagan et al., 2005) and SNLI (Bowman et al., 2015). These examples are only used during OOD evaluation not training.

For evaluation, we follow past work (Hendrycks et al., 2019b) and report the False Alarm Rate at 95\% Recall (FAR95). The FAR95 is the probability that an in-distribution example raises a false alarm, assuming that $95 \%$ of all out-of-distribution exam- ples are detected. Hence a lower FAR95 is better. Partial results are in Figure 4, and full results are in the Appendix.

Previous Models Struggle at OOD Detection. Models without pretraining (e.g., BoW, LSTM word2vec) are often unable to reliably detect OOD examples. In particular, these models' FAR95 scores are sometimes worse than chance because the models often assign a higher probability to out-of-distribution examples than in-distribution examples. The models particularly struggle on 20 Newsgroups (which contains text on diverse topics including computer hardware, motorcycles, space), as their false alarm rates are approximately $100 \%$.

\section{Pretrained Transformers Are Better Detectors.} In contrast, pretrained Transformer models are better OOD detectors. Their FAR95 scores are always better than chance. Their superior detection performance is not solely because the underlying model is a language model, as prior work (Hendrycks et al., 2019b) shows that language models are not necessarily adept at OOD detection. Also note that in OOD detection for computer vision, higher accuracy does not reliably improve OOD detection (Lee et al., 2018), so pretrained Transformers' OOD detection performance is not anticipated. Despite their relatively low FAR95 scores, pretrained Transformers still do not cleanly separate in- and out-of-distribution examples (Figure 5). OOD detection using pretrained Transformers is still far from perfect, and future work can aim towards creating better methods for OOD detection. 


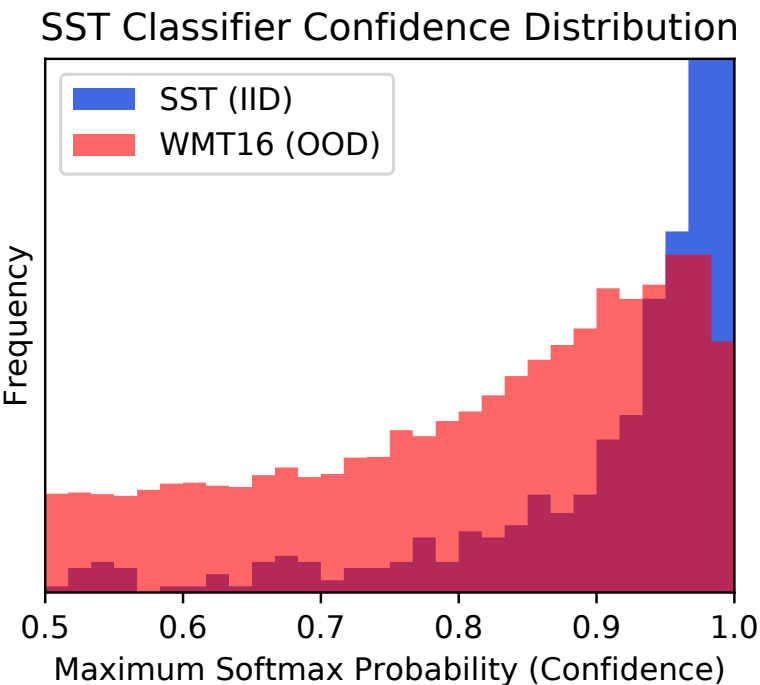

Figure 5: The confidence distribution for a RoBERTa SST-2 classifier on examples from the SST-2 test set and the English side of WMT16 English-German. The WMT16 histogram is translucent and overlays the SST histogram. The minimum prediction confidence is 0.5 . Although RoBERTa is better than previous models at OOD detection, there is clearly room for future work.

\section{Discussion and Related Work}

Why Are Pretrained Models More Robust? An interesting area for future work is to analyze why pretrained Transformers are more robust. A flawed explanation is that pretrained models are simply more accurate. However, this work and past work show that increases in accuracy do not directly translate to reduced IID/OOD generalization gaps (Hendrycks and Dietterich, 2019; Fried et al., 2019). One partial explanation is that Transformer models are pretrained on diverse data, and in computer vision, dataset diversity can improve OOD generalization (Hendrycks et al., 2020) and OOD detection (Hendrycks et al., 2019b). Similarly, Transformer models are pretrained with large amounts of data, which may also aid robustness (Orhan, 2019; Xie et al., 2020; Hendrycks et al., 2019a). However, this is not a complete explanation as BERT is pretrained on roughly 3 billion tokens, while GloVe is trained on roughly 840 billion tokens. Another partial explanation may lie in self-supervised training itself. Hendrycks et al. (2019c) show that computer vision models trained with self-supervised objectives exhibit better OOD generalization and far better OOD detection performance. Future work could propose new self-supervised objectives that enhance model robustness.
Domain Adaptation. Other research on robustness considers the separate problem of domain adaptation (Blitzer et al., 2007; Daumé III, 2007), where models must learn representations of a source and target distribution. We focus on testing generalization without adaptation in order to benchmark robustness to unforeseen distribution shifts. Unlike Fisch et al. (2019); Yogatama et al. (2019), we measure OOD generalization by considering simple and natural distribution shifts, and we also evaluate more than question answering.

Adversarial Examples. Adversarial examples can be created for NLP models by inserting phrases (Jia and Liang, 2017; Wallace et al., 2019), paraphrasing questions (Ribeiro et al., 2018), and reducing inputs (Feng et al., 2018). However, adversarial examples are often disconnected from real-world performance concerns (Gilmer et al., 2018). Thus, we focus on an experimental setting that is more realistic. While previous works show that, for all NLP models, there exist adversarial examples, we show that all models are not equally fragile. Rather, pretrained Transformers are overall far more robust than previous models.

Counteracting Annotation Artifacts. Annotators can accidentally leave unintended shortcuts in datasets that allow models to achieve high accuracy by effectively "cheating" (Cai et al., 2017; Gururangan et al., 2018; Min et al., 2019). These annotation artifacts are one reason for OOD brittleness: OOD examples are unlikely to contain the same spurious patterns as in-distribution examples. OOD robustness benchmarks like ours can stress test a model's dependence on artifacts (Liu et al., 2019a; Feng et al., 2019; Naik et al., 2018).

\section{Conclusion}

We created an expansive benchmark across several NLP tasks to evaluate out-of-distribution robustness. To accomplish this, we carefully restructured and matched previous datasets to induce numerous realistic distribution shifts. We first showed that pretrained Transformers generalize to OOD examples far better than previous models, so that the IID/OOD generalization gap is often markedly reduced. We then showed that pretrained Transformers detect OOD examples surprisingly well. Overall, our extensive evaluation shows that while pretrained Transformers are moderately robust, there remains room for future research on robustness. 


\section{Acknowledgements}

We thank the members of Berkeley NLP, Sona Jeswani, Suchin Gururangan, Nelson Liu, Shi Feng, the anonymous reviewers, and especially Jon Cai. This material is in part based upon work supported by the National Science Foundation Frontier Award 1804794. Any opinions, findings, and conclusions or recommendations expressed in this material are those of the author(s) and do not necessarily reflect the views of the National Science Foundation.

\section{References}

John Blitzer, Mark Dredze, and Fernando Pereira. 2007. Biographies, bollywood, boom-boxes and blenders: Domain adaptation for sentiment classification. In $A C L$.

Samuel R. Bowman, Gabor Angeli, Christopher Potts, and Christopher D. Manning. 2015. A large annotated corpus for learning natural language inference. In $E M N L P$.

Zheng Cai, Lifu Tu, and Kevin Gimpel. 2017. Pay attention to the ending: Strong neural baselines for the roc story cloze task. In $A C L$.

Dallas Card, Michael Zhang, and Noah A. Smith. 2018. Deep weighted averaging classifiers. In FAT.

Daniel Cer, Mona Diab, Eneko Agirre, Inigo LopezGazpio, and Lucia Specia. 2017. SemEval-2017 Task 1: Semantic textual similarity-multilingual and cross-lingual focused evaluation. In SemEval.

Christopher Clark and Matt Gardner. 2018. Simple and effective multi-paragraph reading comprehension. In $A C L$.

Ido Dagan, Oren Glickman, and Bernardo Magnini. 2005. The PASCAL recognising textual entailment challenge. In Machine Learning Challenges Workshop.

Hal Daumé III. 2007. Frustratingly easy domain adaptation. In $A C L$.

Jacob Devlin, Ming-Wei Chang, Kenton Lee, and Kristina Toutanova. 2019. BERT: pre-training of deep bidirectional transformers for language understanding. In NAACL-HLT.

Desmond Elliott, Stella Frank, Khalil Sima' an, and Lucia Specia. 2016. Multi30k: Multilingual englishgerman image descriptions. In $A C L$.

Andrew Emmott, Shubhomoy Das, Thomas G. Dietterich, Alan Fern, and Weng-Keen Wong. 2015. A meta-analysis of the anomaly detection problem.

Shi Feng, Eric Wallace, and Jordan Boyd-Graber. 2019. Misleading failures of partial-input baselines. In $A C L$.
Shi Feng, Eric Wallace, II Grissom, Mohit Iyyer, Pedro Rodriguez, and Jordan Boyd-Graber. 2018. Pathologies of neural models make interpretations difficult. In $E M N L P$.

Adam Fisch, Alon Talmor, Robin Jia, Minjoon Seo, Eunsol Choi, and Danqi Chen. 2019. Proceedings of the 2 nd workshop on machine reading for question answering. In MRQA Workshop.

Daniel Fried, Nikita Kitaev, and Dan Klein. 2019. Cross-domain generalization of neural constituency parsers. In $A C L$.

Prakhar Ganesh, Yao Chen, Xin Lou, Mohammad Ali Khan, Yin Yang, Deming Chen, Marianne Winslett, Hassan Sajjad, and Preslav Nakov. 2020. Compressing large-scale transformer-based models: A case study on BERT. ArXiv, abs/2002.11985.

Matt Gardner, Joel Grus, Mark Neumann, Oyvind Tafjord, Pradeep Dasigi, Nelson F. Liu, Matthew Peters, Michael Schmitz, and Luke S. Zettlemoyer. 2018. AllenNLP: a deep semantic natural language processing platform. In Workshop for NLP Open Source Software.

Justin Gilmer, Ryan P. Adams, Ian J. Goodfellow, David Andersen, and George E. Dahl. 2018. Motivating the rules of the game for adversarial example research. ArXiv, abs/1807.06732.

Suchin Gururangan, Swabha Swayamdipta, Omer Levy, Roy Schwartz, Samuel R. Bowman, and Noah A. Smith. 2018. Annotation artifacts in natural language inference data. In NAACL-HLT.

Zellig S Harris. 1954. Distributional structure. Word.

Ruining He and Julian J. McAuley. 2016. Ups and downs: Modeling the visual evolution of fashion trends with one-class collaborative filtering. In $W W W$.

Dan Hendrycks and Thomas Dietterich. 2019. Benchmarking neural network robustness to common corruptions and perturbations. In ICLR.

Dan Hendrycks and Kevin Gimpel. 2016. Gaussian error linear units (GELUs). arXiv preprint arXiv:1606.08415.

Dan Hendrycks and Kevin Gimpel. 2017. A baseline for detecting misclassified and out-of-distribution examples in neural networks. In ICLR.

Dan Hendrycks, Kimin Lee, and Mantas Mazeika. 2019a. Using pre-training can improve model robustness and uncertainty. ICML.

Dan Hendrycks, Mantas Mazeika, and Thomas G. Dietterich. 2019b. Deep anomaly detection with outlier exposure. ICLR.

Dan Hendrycks, Mantas Mazeika, Saurav Kadavath, and Dawn Song. 2019c. Using self-supervised learning can improve model robustness and uncertainty. In NeurIPS. 
Dan Hendrycks, Norman Mu, Ekin D. Cubuk, Barret Zoph, Justin Gilmer, and Balaji Lakshminarayanan. 2020. AugMix: A simple data processing method to improve robustness and uncertainty. ICLR.

Dan Hendrycks, Kevin Zhao, Steven Basart, Jacob Steinhardt, and Dawn Song. 2019d. Natural adversarial examples. ArXiv, abs/1907.07174.

Sepp Hochreiter and Jürgen Schmidhuber. 1997. Long short-term memory. In Neural Computation.

Robin Jia and Percy Liang. 2017. Adversarial examples for evaluating reading comprehension systems. In EMNLP.

Zhenzhong Lan, Mingda Chen, Sebastian Goodman, Kevin Gimpel, Piyush Sharma, and Radu Soricut. 2020. ALBERT: a lite BERT for self-supervised learning of language representations. In ICLR.

Ken Lang. 1995. NewsWeeder: Learning to filter Netnews. In ICML.

Kimin Lee, Honglak Lee, Kibok Lee, and Jinwoo Shin. 2018. Training confidence-calibrated classifiers for detecting out-of-distribution samples. In ICLR.

Zhuohan Li, Eric Wallace, Sheng Shen, Kevin Lin, Kurt Keutzer, Dan Klein, and Joseph E Gonzalez. 2020. Train large, then compress: Rethinking model size for efficient training and inference of transformers. ArXiv, abs/2002.11794.

Nelson F Liu, Roy Schwartz, and Noah A Smith. 2019a. Inoculation by fine-tuning: A method for analyzing challenge datasets. In $N A A C L$.

Yinhan Liu, Myle Ott, Naman Goyal, Jingfei Du, Mandar Joshi, Danqi Chen, Omer Levy, Mike Lewis, Luke Zettlemoyer, and Veselin Stoyanov. 2019b. RoBERTa: A robustly optimized BERT pretraining approach. ArXiv, abs/1907.11692.

Andrew L Maas, Raymond E Daly, Peter T Pham, Dan Huang, Andrew Y Ng, and Christopher Potts. 2011. Learning word vectors for sentiment analysis. In $A C L$.

Julian J. McAuley, Christopher Targett, Qinfeng Shi, and Anton van den Hengel. 2015. Image-based recommendations on styles and substitutes. In SIGIR.

Tomas Mikolov, Ilya Sutskever, Kai Chen, Greg S Corrado, and Jeff Dean. 2013. Distributed representations of words and phrases and their compositionality. In NIPS.

Sewon Min, Eric Wallace, Sameer Singh, Matt Gardner, Hannaneh Hajishirzi, and Luke Zettlemoyer. 2019. Compositional questions do not necessitate multi-hop reasoning. In $A C L$.

Aakanksha Naik, Abhilasha Ravichander, Norman Sadeh, Carolyn Rose, and Graham Neubig. 2018. Stress test evaluation for natural language inference. In COLING.
A. Emin Orhan. 2019. Robustness properties of facebook's ResNeXt WSL models. ArXiv, abs/1907.07640.

Jeffrey Pennington, Richard Socher, and Christopher D. Manning. 2014. GloVe: Global vectors for word representation. In EMNLP.

Joaquin Quionero-Candela, Masashi Sugiyama, Anton Schwaighofer, and Neil D. Lawrence. 2009. Dataset shift in machine learning.

Marco Tulio Ribeiro, Sameer Singh, and Carlos Guestrin. 2018. Semantically equivalent adversarial rules for debugging NLP models. In $A C L$.

Victor Sanh, Lysandre Debut, Julien Chaumond, and Thomas Wolf. 2019. DistilBERT, a distilled version of bert: smaller, faster, cheaper and lighter. In NeurIPS EMC ${ }^{2}$ Workshop.

Sheng Shen, Zhen Dong, Jiayu Ye, Linjian Ma, Zhewei Yao, Amir Gholami, Michael W Mahoney, and Kurt Keutzer. 2020. Q-BERT: Hessian based ultra low precision quantization of BERT.

Richard Socher, Alex Perelygin, Jean Wu, Jason Chuang, Christopher D Manning, Andrew Ng, and Christopher Potts. 2013. Recursive deep models for semantic compositionality over a sentiment treebank. In $E M N L P$.

Antonio Torralba and Alexei A. Efros. 2011. Unbiased look at dataset bias. CVPR.

Ashish Vaswani, Noam Shazeer, Niki Parmar, Jakob Uszkoreit, Llion Jones, Aidan N Gomez, Łukasz Kaiser, and Illia Polosukhin. 2017. Attention is all you need. In NIPS.

Eric Wallace, Shi Feng, Nikhil Kandpal, Matthew Gardner, and Sameer Singh. 2019. Universal adversarial triggers for attacking and analyzing NLP. In EMNLP.

Alex Wang, Amanpreet Singh, Julian Michael, Felix Hill, Omer Levy, and Samuel R. Bowman. 2019. GLUE: A multitask benchmark and analysis platform for natural language understanding. In ICLR.

John Wieting, Mohit Bansal, Kevin Gimpel, and Karen Livescu. 2016. Towards universal paraphrastic sentence embeddings. In ICLR.

Adina Williams, Nikita Nangia, and Samuel R Bowman. 2018. A broad-coverage challenge corpus for sentence understanding through inference. In NAACL-HLT.

Thomas Wolf, Lysandre Debut, Victor Sanh, Julien Chaumond, Clement Delangue, Anthony Moi, Pierric Cistac, Tim Rault, Rémi Louf, Morgan Funtowicz, and Jamie Brew. 2019. HuggingFace's Transformers: State-of-the-art natural language processing. ArXiv, abs/1910.03771. 
Cihang Xie and Alan L. Yuille. 2020. Intriguing properties of adversarial training at scale. In ICLR.

Qizhe Xie, Eduard H. Hovy, Minh-Thang Luong, and Quoc V. Le. 2020. Self-training with noisy student improves imagenet classification. In CVPR.

Dani Yogatama, Cyprien de Masson d'Autume, Jerome Connor, Tomás Kociský, Mike Chrzanowski, Lingpeng Kong, Angeliki Lazaridou, Wang Ling, Lei Yu, Chris Dyer, and Phil Blunsom. 2019. Learning and evaluating general linguistic intelligence. ArXiv, abs/1901.11373.

Sheng Zhang, Xiaodong Liu, Jingjing Liu, Jianfeng Gao, Kevin Duh, and Benjamin Van Durme. 2018. ReCoRD: bridging the gap between human and machine commonsense reading comprehension. arXiv, $\mathrm{abs} / 1810.12885$. 